Vietnam Journal of Mechanics, VAST, Vol.29, No. 3 (2007), pp. $397-413$

Special Issue Dedicated to the Memory of Prof. Nguyen Van Dao

\title{
ON THE TECHNICAL DIAGNOSTICS OF BALL-BEARING BASED ON MODELLING OF ROTOR-BEARING SYSTEMS BY VIBRATION METHOD
}

\author{
Nguyen CaO Menh \\ Institute of Mechanics, VAST
}

\begin{abstract}
In this paper, after analysing deformations of loading ball-bearing it is able to investigate vibrations of rotor-bearing system as the system with non-linear and periodically varying stiffness. The modelling of the systems is depent on properties of the shaft (rigid or flexible) and on measurement techniques for system vibrations (by eddy current proximity probe for rotating shaft or on non-rotating parts of the rotor-bearing systems) it can be described by a single degree-of-freedom or two-degree-of freedom nonlinear systems subjected to parameteric and external excitations. Therefore it is possible to obtain different characteristics of system vibrations with respect to different defects of ball-bearing. These symptoms help us to identify and estimate the bearing quality by measurement and analysis of system vibrations.
\end{abstract}

\section{INTRODUCTION}

As we know, vibrations of machine is one of important signals for diagnostics of machinery condition. If the systems consist of rotating part (rotor) and supported-bearings, then it is called as rotor-bearing systems. The rolling element bearings are a type of bearings, its rolling element can be balls, rollers, tapered rollers, spherical rollers or needles. These bodies are positioned within a cage which holds them at a set spacing. In this paper the ball-bearing in a rotor-bearing system will be considered for some different types of rotors and the technical condition of the ball-bearings are estimated by processing and analysis of vibration data, which can be measured for the systems. Loading of the bearing leads to movement between the races. Their mathematical relationship must be known in order to be able to determine the influence of ball-bearings on the dynamics of rotors. Some defects of the ball-bearings are simulated for estimating its influences on vibrations of the system. It is necessary to notice that the symptoms for assessment of the ball-bearing conditon are depent on the techniques of vibration measurment, for example, relative displacement between the journal and bearing (measured by proximity probe) or vibrations of the bearings (measured by accelerometers).

In the below, we introduce breafly some results published from [4-8] and give some new supplementary archivements.

\section{THE MODELLING OF ROTOR-BEARING SYSTEMS}

The rotor-bearing systems can be modeled as in the Fig. 1, where $M$ - rotor mass, $m$ - eccentric mass and $C, K$ are damping and stiffness respectively, generated by bearings 
under loading. If the rotor with shaft is regarded as a rigid body then the system can be considered as a system with single degree of freedom.



Fig. 1

\subsection{Analysing rotating motion of a ball-bearing}

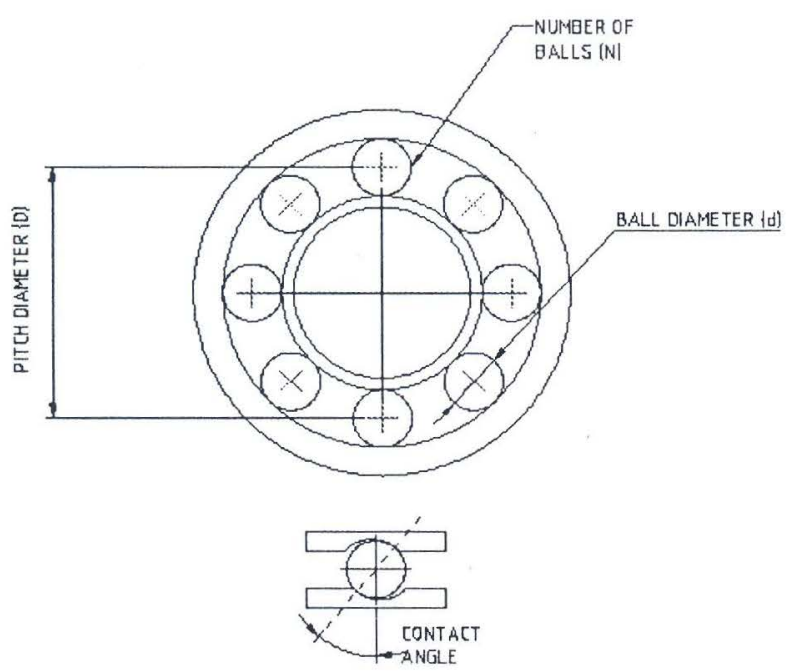

Fig. 2

Consider a single ball-bearing (Fig. 2), which consists of inner race, $N$ balls and outer race. In the rotor-bearing system, the inner race rotates together with the shaft of the system and outer race is not moved. On the basis of analyzing revolution mechanism of the balls-bearing $[1,2,3]$ we can obtain the following results:

- The contact frequency $f_{i}$ of one point on inner race with $N$ ball:

$$
f_{i}=\frac{1}{2} f N\left(1+\frac{d}{D} \cos \alpha\right)
$$

where $\alpha$ is contact angle of a ball with races.

- The contact frequency $f_{e}$ of one point on outer race with $\mathrm{N}$ ball:

$$
f_{e}=\frac{1}{2} f N\left(1-\frac{d}{D} \cos \alpha\right) .
$$

- The contact frequency $f_{b}$ of one point on a ball with inner race or outer race

$$
f_{b}=\frac{f}{2} \frac{D}{d}\left[1-\left(\frac{d}{D}\right)^{2} \cos ^{2} \alpha\right],
$$


where $f$-revolutions per second of inner race (shaft), $N$ - number of balls, $D$ - pitch diameter, $d$ - ball diameter, $\alpha$ - contact angle.

From above results, we will have the frequencies of forces generated by different defects of ball-bearing.

If there is one defect in the inner race, then the force appears in the system with frequency $f_{i}$ given by the formula (2.1). Similarly, the frequency of force occured by one defect in the outer race, is $f_{e}$ given by the formula (2.2). On the other hand, if one defect is occurred in one ball, then the frequency of force generated by the defect is $f_{b}$ presented by the formula (2.3).

\subsection{Stiffness of ball element bearings}

At first, on the basis of analysis of bearing deformations $[3,4]$ we have:

$$
F=F_{s}+F_{d}=\left[N(1.2)^{-\frac{3}{2}} d^{\frac{1}{2}}\right]\left(x_{0}+x\right)^{\frac{3}{2}}
$$

where $F$ - total load (both static and dynamic), $F_{s^{-}}$static load, $F_{d}$ - dynamic load, $x$ dynamic deformation, $x_{0}$ - static deformation under load $\mathrm{F}_{s}$, and then

$$
F_{s}=\left[N(1.2)^{-\frac{3}{2}} d^{\frac{1}{2}}\right] x_{0}^{\frac{3}{2}}
$$

From (2.4), it is able to obtain the following expressions:

$$
F=F_{s}+F_{d}=\left[N(1.2)^{-\frac{3}{2}} d^{\frac{1}{2}}\right] x_{0}^{\frac{3}{2}}(1+u)^{\frac{3}{2}}=F_{s}(1+u)^{\frac{3}{2}}
$$

where $u=x / x_{0}$. Expand (2.6) and taking into account of (2.5), we have:

$$
F_{d}=F_{s}\left(\frac{3}{2} u+\frac{3}{8} u^{2}-\frac{1}{16} u^{3}+\ldots\right)
$$

Notice that $u=x / x_{0}$ then the stiffness coefficient for respective linearized system:

$$
K=\frac{3}{2} \frac{F_{s}}{x_{0}}=\frac{3}{2} \frac{M g}{x_{0}}
$$

and the formula (2.7) is in the form:

$$
F_{d}=K x_{0}\left(u+\frac{1}{4} u^{2}-\frac{1}{24} u^{3}+\ldots\right)
$$

Here $K$ is measured in $N / \mu \mathrm{m}, M$-in $\mathrm{kg}, g-$ in $\mathrm{m} / \mathrm{s}^{2}, d-$ in $\mathrm{mm}$ and $x_{0}-$ in $\mu \mathrm{m}$.

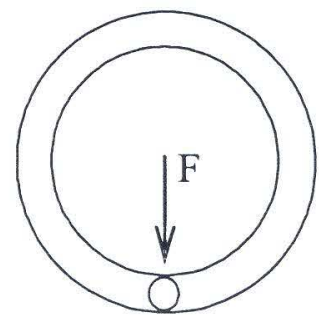

a)

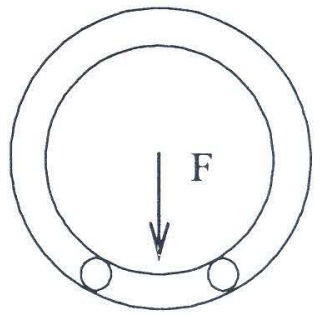

b)

Fig. 3

However, if we are interested in the vertical direction then the stiffness of the ballbearing is changed periodically beacause of the revolution of the balls in the bearing. If 
one ball is located in the lowest position (Fig. 3a) the stiffness is calculated in the form (2.8), but if the balls are located as in the Fig. 3b, then the stiffness is smaller. Therefore the stiffness of ball-bearings is changed periodically with the frequency $f_{e}$ in the formula (2.2) and has the form

$$
K(t)=K\left(1-\varepsilon \cos \omega_{e} t\right)
$$

where $\omega_{e}=2 \pi f_{e}, \varepsilon$ is a small parameter, and from the formula (2.9) the restoration force becomes:

$$
F_{d}=K x_{0}\left(1-\varepsilon \cos \omega_{e} t\right)\left(u+\frac{1}{4} u^{2}-\frac{1}{24} u^{3}+\ldots\right)
$$

In the below, we will consider some kinds of simulated rotor-bearing systems, which are depent on properties of roror shafts and measurement techniques of vibration.

\section{RELATIVE VIBRATION OF RIGID ROTOR JOURNAL $[4,5,6]$}

\subsection{Motion Equation}

In this section the vibrations between journal of rigid rotor and bearing-support are investigated, then it has a modelling in the Fig. 1 and is described by single-degree-offreedom system.

$$
M \ddot{x}+C \dot{x}+F_{d}(x)=F(t)
$$

where $x$-displacement of the shalf in vertical direction, $M, C$ - constans of mass, damping (because $m<<M$ then $\mathrm{m}$ is negleted), $F(t)$ - external forces, which consist of centrifugal force of unbalance mass and different forces generated by defects of ball- bearings,... In practice the obtained solutions can be compared with vibrations measured by proximity probes for identication of bearing defects in rotor-bearing system.

Substituting (2.11) into (3.1) and taking into account of (2.8) we can get:

$$
\ddot{u}+\frac{C}{M} \dot{u}+\frac{3}{2} \frac{g}{x_{0}}\left[1-\varepsilon \cos \left(\omega_{e} t\right)\right]\left(u+\frac{1}{4} u^{2}-\frac{1}{24} u^{3}\right)=\frac{1}{M x_{0}} F(t)
$$

or

$$
\ddot{u}+2 h \dot{u}+\omega_{n}^{2}\left[1-\varepsilon \cos \left(\omega_{e} t\right)\right]\left(u+\frac{1}{4} u^{2}-\frac{1}{24} u^{3}\right)=\frac{1}{M x_{0}} F(t)
$$

where

$$
h=\frac{C}{2 M}, \quad \omega_{n}^{2}=\frac{1}{2 x_{0}}, \quad u=x / x_{0}
$$

and $x_{0}$ is given by (2.5), that means:

$$
x_{0}=1.2 d^{-1 / 3}\left[\frac{M g}{N}\right]^{2 / 3}
$$

\subsection{External forces}

The external forces applied to the rotor-bearing systems consist of the folowing ones:

Inertial cetrifugal force is generated by unbalance mass $m$. If vertical vibrations are investigated then this force is in the form:

$$
F_{I}=m r(2 \pi f)^{2} \sin (2 \pi f t)
$$

where $f$-speed of rotor revolution per second. 
If the ball-bearing has a defect on inner race then in the process of operation of rotorbearing system the impact is rised and can be able to express as follows:

$$
F_{v 1}=\sum_{i=1}^{\infty} H_{1 i} \delta\left(t-i T_{1}\right)
$$

where $F_{v 1}$ is impact force of inner race defect, $H_{1 i}$ - intensity of the force, $\delta$ - Dirac function, $T_{1}$ - period of impact, $T_{1}=\left(f_{i}\right)^{-1}$ and $f_{i}$ is given by $(2.1)$.

It is similar to the case of defect on outer race:

$$
F_{v 2}=\sum_{i=1}^{\infty} H_{2 i} \delta\left(t-i T_{2}\right)
$$

where $T_{2}=\left(f_{e}\right)^{-1}$, and $f_{e}$ is given by (2.2).

If there is a brokken ball in the bearing then the impact force has the period $T_{3}=$ $\left(f_{b}\right)^{-1}, f_{b}$ given by (2.3), and expressed by the formula:

$$
F_{v 3}=\sum_{i=1}^{\infty} H_{3 i} \delta\left(t-i T_{3}\right)
$$

The forces $F_{I}, F_{v 1}, F_{v 2}, F_{v 3}$ are typical ones generated from rotor-bearing system. Therefore, in right side of the equation (3.2) function $F(t)$ can be in the form:

$$
F(t)=F_{I}(t)+F_{v 1}(t)+F_{v 2}(t)+F_{v 3}(t)
$$

In the expression (3.9), $F_{I}$ is alway existed, because there is usually a umbalance mass of rotor, and the other forces $F_{v 1}, F_{v 2}, F_{v 3}$ could not be existed at the same time. In computation these functions $\mathrm{F}_{k}(\mathrm{t})$ can be expanded in Fourier series and some relevant terms can be taken:

$$
F_{k}=2 \pi f_{k} H_{k 0}+\sum_{j=1}^{\infty} 4 \pi f_{k} H_{k j} \sin \left(2 \pi j f_{k} t\right)
$$

where $k=I, v 1, v 2, v 3$, and $f_{k}=f, f_{i},{ }^{\bullet} f_{e}, f_{b}$, respectively.

Notice that thís problem will be investigated in frequency domain then some parameters such as $\mathrm{H}_{k j}, \varepsilon, \ldots$ could be chosen and in numerical calculation we choose only some terms of sum (3.10).

\subsection{The results of calculation for some cases}

Consider a rotor-bearing system, for example, using ball-bearings 36212 with the following parameters:

- Rotor mass $1100 \mathrm{~kg}$, uniformly distributed on two bearings, then $M=550[\mathrm{~kg}]$.

- Speed of revolution $725 \mathrm{rpm}$, then $\mathrm{f}=12.08 \mathrm{rev} / \mathrm{s}$.

- Eccentric mass $m=0.2[\mathrm{~kg}]$

- Eccentric radius $\mathrm{r}=0.4[\mathrm{~m}]$

- Number of balls $N=10$

- Ball diameter $\mathrm{d}=15.88[\mathrm{~mm}]$

- Pitch diameter $\mathrm{D}=85.1[\mathrm{~mm}]$

Contact angle $\alpha=12^{0}$.

For this example it is able to calculate the following parameters:

$f_{n}=108.5 \mathrm{~Hz}\left(\omega_{n}=2 \pi f_{n}\right), f_{i}=71.42 \mathrm{~Hz}, f_{e}=49.37 \mathrm{~Hz}$ 
$f_{b}=33.48 \mathrm{~Hz}, f=12.08 \mathrm{~Hz}, x_{0}=31.64 \mu \mathrm{m}$

Some cases are follows:

a) The stiffness of bearings is constant and $\mathrm{F}(\mathrm{t})=\mathrm{F}_{I}(\mathrm{t})+\mathrm{F}_{v 1}(\mathrm{t})$, the equation (3.2) has the form:

$$
\ddot{u}+2 h \dot{u}+\omega_{n}^{2} u=\frac{1}{M x_{0}} F(t)=\frac{1}{M x_{0}}\left[F_{I}(t)+F_{v 1}(t)\right]
$$

b) The stiffness of bearings is periodically varying

$$
K(t)=K\left(1-\cos \omega_{e} t\right)
$$

and $F(t)=F_{I}(t)+F_{v 1}(t)$, the motion equation is governed:

$$
\ddot{u}+2 h \dot{u}+\omega_{n}^{2}\left[1-\varepsilon \cos \left(\omega_{e} t\right)\right]=\frac{1}{M x_{0}}\left[F_{I}(t)+F_{v 1}(t)\right]
$$

c) The stiffness of bearing is nonlinear and $\mathrm{F}(\mathrm{t})=\mathrm{F}_{I}(\mathrm{t})+\mathrm{F}_{v 1}(\mathrm{t})$, the equation (3.2) can get:

$$
\ddot{u}+2 h \dot{u}+\omega_{n}^{2}\left(u+\frac{1}{4} u^{2}-\frac{1}{24} u^{3}\right)=\frac{1}{M x_{0}}\left[F_{I}(t)+F_{v 1}(t)\right]
$$

d) The stiffness of bearing is periodically varying, nonlinear and $F(t)=F_{I}(t)+F_{v 1}(t)$, the equation (3.2) can get:

$\ddot{u}+2 h \dot{u}+\omega_{n}^{2}\left[1-\varepsilon \cos \left(\omega_{e} t\right)\right]\left(u+\frac{1}{4} u^{2}-\frac{1}{24} u^{3}\right)=\frac{1}{M x_{0}}\left[F_{I}(t)+F_{v 1}(t)\right]$

e) It is similar for the other cases, for example, there is a defect on outer race, then the equation (3.2) is:

$$
\ddot{u}+2 h \dot{u}+\omega_{n}^{2}\left[1-\varepsilon \cos \left(\omega_{e} t\right)\right]\left(u+\frac{1}{4} u^{2}-\frac{1}{24} u^{3}\right)=\frac{1}{M x_{0}}\left[F_{I}(t)+F_{v 2}(t)\right]
$$

The Vibrations and their spectra are expressed in figures 4 and 5 for the cases a)-e).

\section{VIBRATION OF RIGID ROTOR [7]}

In many practical cases, if it is only possible to measure vibrations on non-rotating parts of the rotor-bearing system such as on bearing cages, foundations,... by direct transdusers (such as accelerometors) and using the data for analysis, then it is necessary to change the mathematical modelling of the system. If the vertical vibrations will be considered, then the system modelling consists of two masses, they are the mass of the shaft and the rotor placed on ball-bearings and the mass of bearing cages, supported parts and foundations (non-rotating parts) on elastic bases. Therefore, in this case, the system modelling is considered as two-degree-of-freedom system of vibration. The main problem is to assess the parameters of the system and the excited loads and find out vertical vibrations of the non-rotating parts for comparison with the analysis of measurement data. 

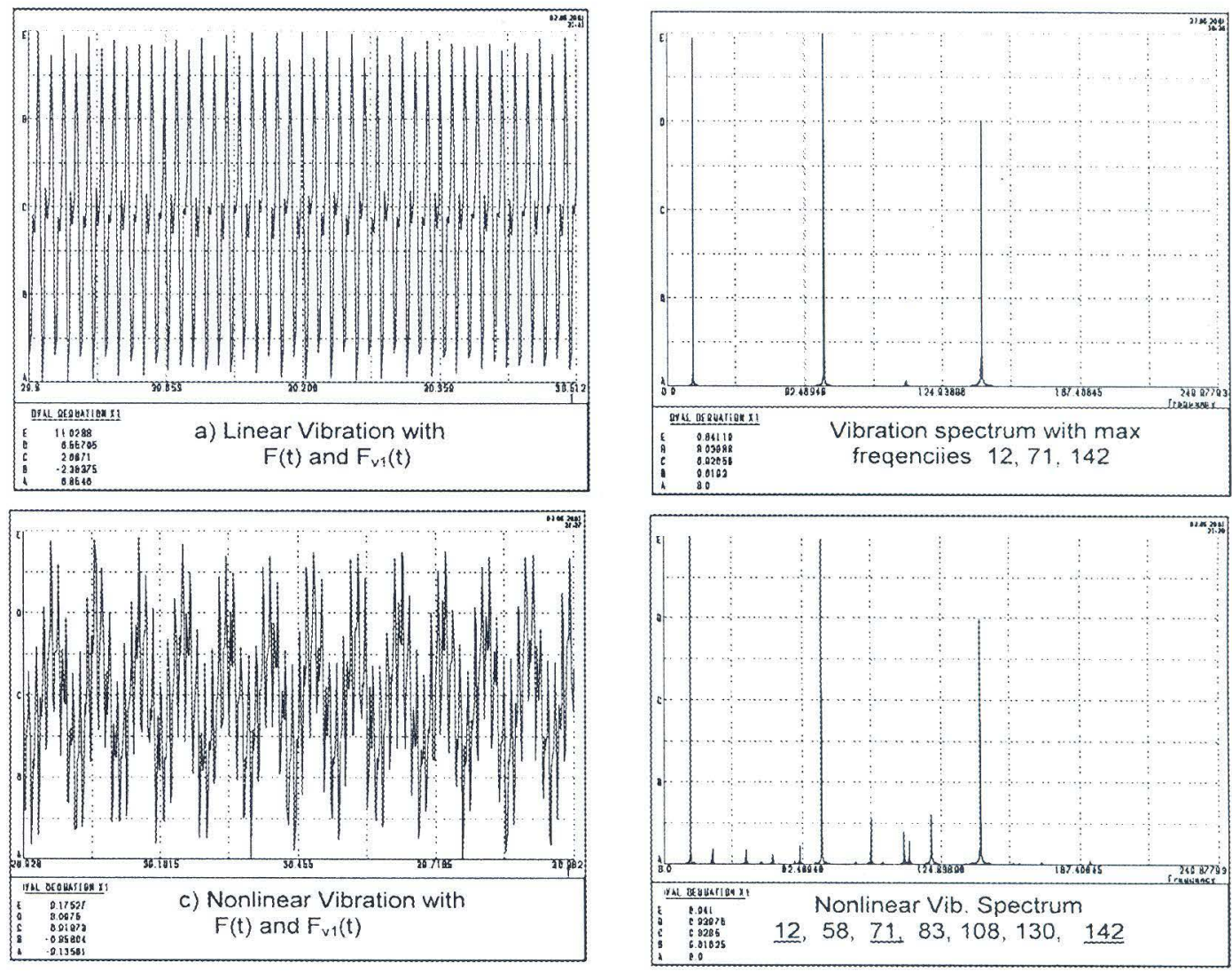

Fig. 4. Vibrations and Specra for the cases a) and c)

\subsection{Motion equations}

Consider the vibration system consisted of a rigid rotor, two ball-bearings on supported parts (machine and foundation). The mass of rotor and shaft is denoted by $m$, the stiffness of ball-bearings in vertical direction is $k_{1}$, the mass of supported parts is $\mathrm{M}$ on elastic base with stiffness $k_{2}$. Assume that unbalance mass of rotor is $m_{0}$ with the distance $\mathrm{r}$ from shaft line. The modelling of vertical vibrations of the system with rotating rotor is given on the Fig. 6. From the modelling of vibration system (Fig. 6) the motion equations are governed by:

$$
\begin{aligned}
& m \ddot{x}_{1}+c_{1}\left(\dot{x}_{1}-\dot{x}_{2}\right)+k_{1}\left(x_{1}-x_{2}\right)=F(t) \\
& M \ddot{x}_{2}+\left(c_{1}+c_{2}\right) \dot{x}_{2}+\left(k_{1}+k_{2}\right) x_{2}-c_{1} \dot{x}_{1}-k_{1} x_{1}=0
\end{aligned}
$$

The rotor is supported on two ball-bearings, then $\mathrm{k}_{1}=2 \mathrm{~K}$, in which $K$ is stiffness of one ball-bearing, calculated by the formula [4]:

$$
K=(1.25) N^{2 / 3}(0.5 m g d)^{1 / 3}[N / \mu m]
$$

The other coefficients in the equation (4.1) are found by calculation or experiment. Consider a single ball-bearing, which consists of inner race, $N$ balls and outer race. In this case, following $[3,4]$, the stiffness of ball-bearings can be expressed by periodically timevarying function as follows:

$$
k_{1}=2 K\left[1-\varepsilon \cos \left(\omega_{e} t\right)\right]
$$



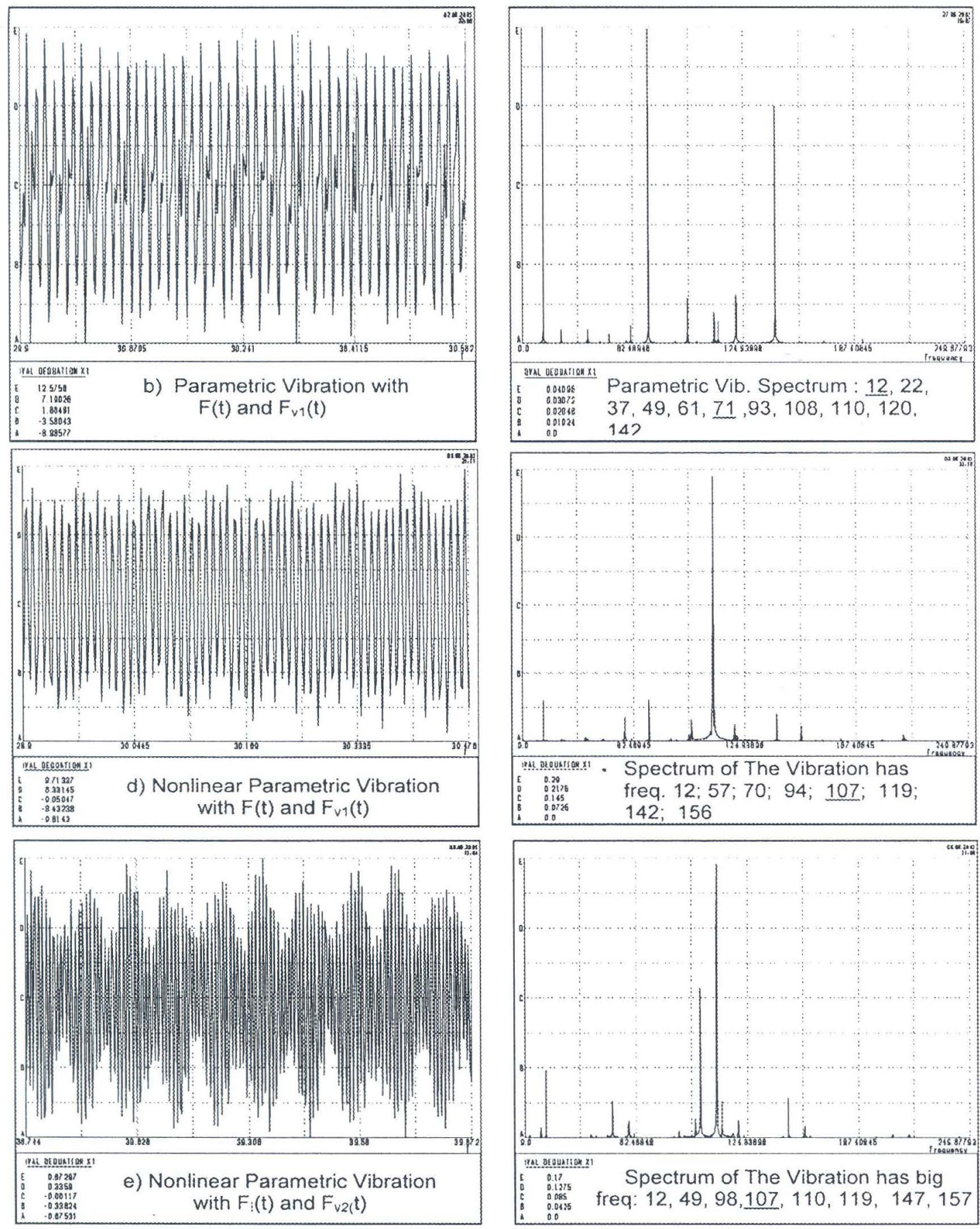

Fig. 5. Vibrations and Specra for the cases b), d) and e)

The equation (2.1) has now the form:

$$
\begin{aligned}
& m \ddot{x}_{1}+c_{1}\left(\dot{x}_{1}-\dot{x}_{2}\right)+2 K\left[1-\varepsilon \cos \left(\omega_{e} t\right)\right]\left(x_{1}-x_{2}\right)=F(t) \\
& M \ddot{x}_{2}+\left(c_{1}+c_{2}\right) \dot{x}_{2}+2 K\left[1-\varepsilon \cos \left(\omega_{e} t\right)\right]\left(x_{2}-x_{1}\right)+k_{2} x_{2}-c_{1} \dot{x}_{1}=0
\end{aligned}
$$




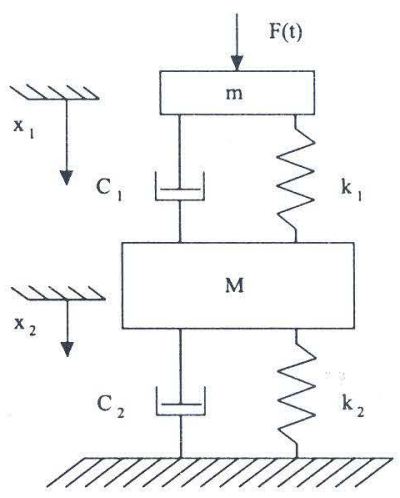

Fig. 6

This is a system of two differential equations of second order with parametric excitation and external forces. The system has two natural frequencies and the responses of the system for this case is more complicated.

\subsection{External forces}

In this case, the dynamic loads applied on the system are similar as previous section, but here these forces are in right hand of the first equation (4.4) and have the form:

$$
F(t)=F_{I}(t)+F_{v 1}(t)+F_{v 2}(t)+F_{v 3}(t)
$$

where the right forces are given by formulae (3.5) - (3.8).

\subsection{Some results of calculation.}

Consider a rotor-bearing system, for example, using ball-bearings 36309, with the following parameters:

- Rotor mass $m=2000[\mathrm{~kg}]$

- Non-rotating parts mass $M=8000[\mathrm{~kg}]$

- Eccentric mass $m_{0}=0.5[\mathrm{~kg}]$

- The distance from $m_{0}$ to the shaft line $r=0.5[\mathrm{~m}]$

- Number of balls $N=12$

- Ball diameter $d=17.46[\mathrm{~mm}]$

- Pitch diameter $D=72.5[\mathrm{~mm}]$

- Contact angle $\alpha=0$.

The other parameters are given in detail computation of different cases

1) The parameter $k_{1}$ is calculated from above formulae, and take $k_{2}=18000 \mathrm{~N} / \mathrm{cm}$, speed of revolution $f=50 \mathrm{~Hz}$, and in diagnosis of the ball-bearing we are interested in frequencies of response of the system, therefore, the other parameters can be chosen. In this case, natural frequencies $f_{01}$ and $f_{02}$ are found by computation

$$
f_{01}=21.24 \mathrm{~Hz} ; \quad f_{02}=107.86 \mathrm{~Hz}
$$

After solving the differential equations (4.4) in the case where there are parametric excitation and faults of the ball-bearings, we obtain two spectral densities of vibrations of the rotating shaft (Fig. 7) and non-rotating parts (foundation, Fig. 8) with frequencies of spectral peaks listed in the Table 1 
From this table, we can see that the vibration spectrum of foundation gives out more poor information of vibration frequency than vibration spectrum of rotating shaft.

Table 1

\begin{tabular}{|c|c|c|c|c|c|c|c|c|}
\hline $\begin{array}{c}\text { Vib. spectrum } \\
\text { of shaft }\end{array}$ & 21.24 & 50. & 110.46 & 116.58 & 177.41 & 195.37 & 221.2 & 227.2 \\
\hline $\begin{array}{c}\text { Vib. spectrum } \\
\text { of foundation }\end{array}$ & 21.24 & 50. & 110.46 & 116.58 & & & & \\
\hline
\end{tabular}

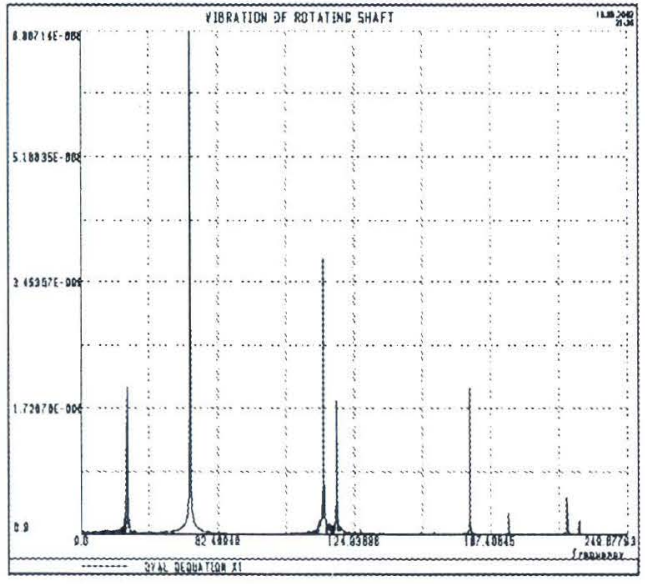

Fig. \%. Spectrum of shaft vibration with fauls of ball-bearing $f=50 \mathrm{~Hz}$

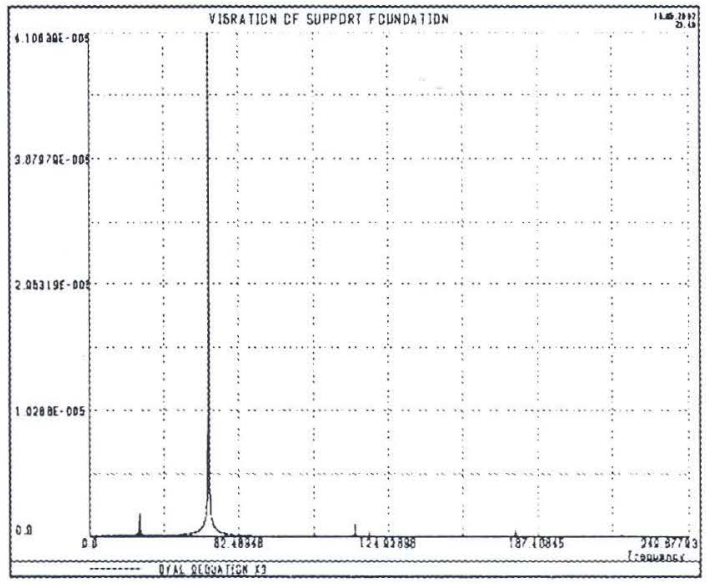

Fig. 8. Spectrum of foundation Vibration with fauls of ball-bearing $f=50 \mathrm{~Hz}$

2) Consider the case where the speed of rotor rotation is less than the first natural frequency, $\mathrm{f}=20 \mathrm{~Hz}$, then the spectra of the rotating shaft vibration and foundation vibration are not much different. Therefore, in this case we can use the data of vibration measurement of non-rotating parts for diagnosis of the ball-bearing. The computational results are given on the Fig. 9 and Fig. 10 with spectral peaks in Table 2.

Table 2

\begin{tabular}{|c|c|c|c|}
\hline Vib.Spectrum of Shaft & 20.0 & 107.6 & 197.8 \\
\hline Vib.Spectrum of foundation & 20.0 & 107.6 & 197.8 \\
\hline
\end{tabular}

Hence, for assessment of the ball-bearing quality by using data of vibration measurement on non-rotating parts it is necessary to notice that the modelling of the rotor-bearing system is two-degree-of-freedom system with parametric excitation and external forces, and the rotation speed of the rotor has great effect on the information of measurement data. Therefore, the relative vibration measurement between journal and bearing is better for diagnosis of ball-bearing quality. 


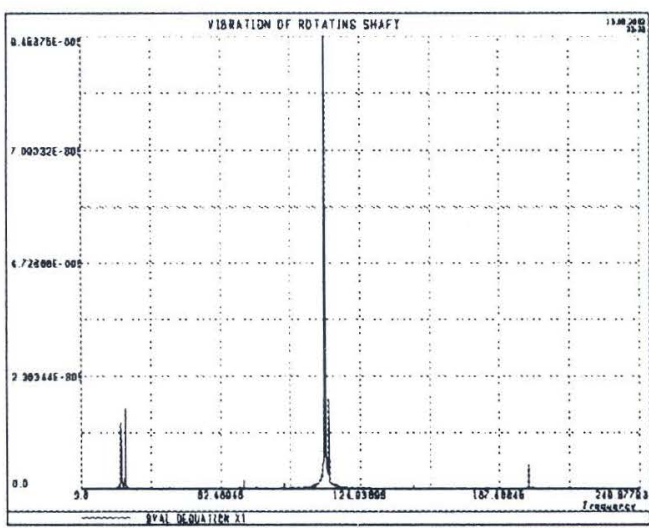

Fig. 9. Spectrum of shaft vibration with fauls of ball-bearing $f=20 \mathrm{~Hz}$

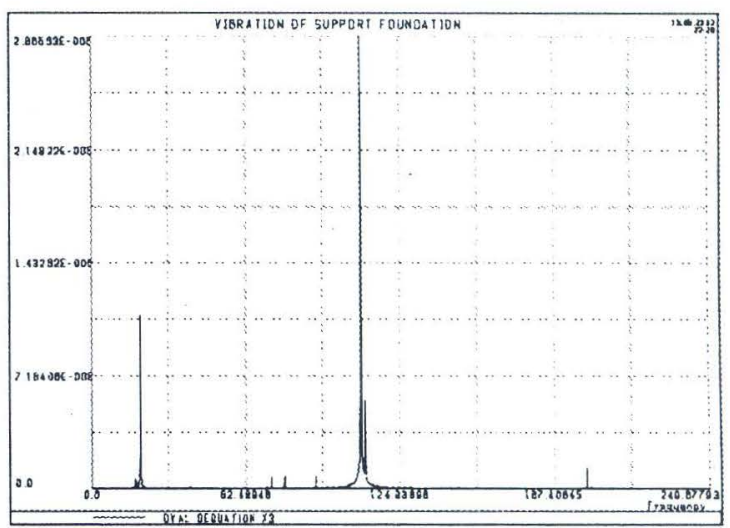

Fig. 10. Spectrum of foundation Vibration with fauls of ball-bearing $f=20 \mathrm{~Hz}$

\section{VIBRATIONS OF JEFFCOTT ROTOR ON BALL-BEARINGS [8]}

In this section, the systems consisted of Jeffeott rotor and ball-bearings are investigated (Fig. 11). The Jeffeott rotor is regarded as the most simple rotor model consisted of a massless shaft, at whose center is a fixed rigid circular disc and which is supported in rigid bearings. The center of mass $\mathrm{S}$ can be coincident with the shaft center IV or can have a radial offset, or eccentricity e [1]. But in our case, the bearings are flexible, and bearing stiffness used in the mathematical modeling is regarded as periodically varying with time and nonlinear. On the basis of this property and the shaft elastic deformation it is able to explain several phenomena obtained from measurement vibrations of the system.and then the technical conditions of the ball-bearings and the system can be diagnosed.

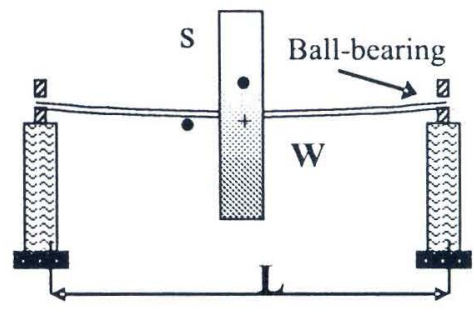

Fig. 11

\subsection{Motion Equations}

Assume that the shaft stiffness is $\mathbf{k}_{S}$ and its damping is $\mathbf{d}_{s}$ (Fig. 1). For ball-bearing, its stiffness is $\mathbf{k}_{B}$ and the damping is $\mathbf{d}_{B}$.

The modelling of the system is described in the Fig. 12. The distance between two ball-bearings is $L$, then $k_{S}$ is computated by the formula:

$$
k_{S}=(48 E J) / L^{3}
$$

where $E$ - Young's modulus, $J$ - area moment of inertia of shaft cross section. In this case, we restrict ourseft to investigate periodically varying stiffness, then the bearing stiffness 
is calculated by following formula $[3,4]$

$$
\begin{gathered}
k_{B}=K_{B}\left[1-\varepsilon \cos \left(\omega_{e} t\right)\right] \\
K_{B}=(1.25) N^{2 / 3} d^{1 / 3}(M g / 2)^{1 / 3}[N / \mu m]
\end{gathered}
$$

where $N$ - number of balls, $d$ - ball diameter (in mm), $M$ - mass of rotor, $m$-mass of inner race (in $\mathrm{kg}$ ) and $g$-gravity acceleration (in $\mathrm{m} / \mathrm{s}^{2}$ ), $\varepsilon$ is small dimensionless value, $\omega_{e}=2 \pi f_{e}$ with $f_{e}$ is given by $(2.2)$.

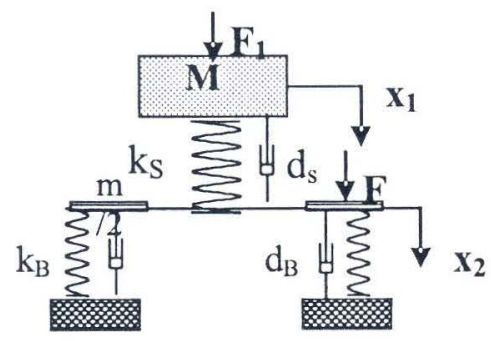

Fig. 12

The motion equations of the system for vertical vibrations are governing as follows:

$$
\begin{aligned}
& M \ddot{x}_{1}+d_{s}\left(\dot{x}_{1}-\dot{x}_{2}\right)+k_{s}\left(x_{1}-x_{2}\right)=F_{1}(t) \\
& m \ddot{x}_{2}+2 d_{B} \dot{x}_{2}-d_{s}\left(\dot{x}_{1}-\dot{x}_{2}\right)+ \\
& \quad+2 k_{B} x_{2}-k_{s}\left(x_{1}-x_{2}\right)=F(t)
\end{aligned}
$$

where $k_{S}, k_{B}$ are given by formulae (5.1),(5.2),(5.3), the value $d_{B}$ is, in general, small and determined by experiment. The equation system (5.4) with periodically time-varying stiffness $\mathrm{k}_{B}$ is excited by external forces. If $\varepsilon$ is too small, we can take $k_{B}=K_{B}$ and the system (5.4) becomes a system with constant coefficients.

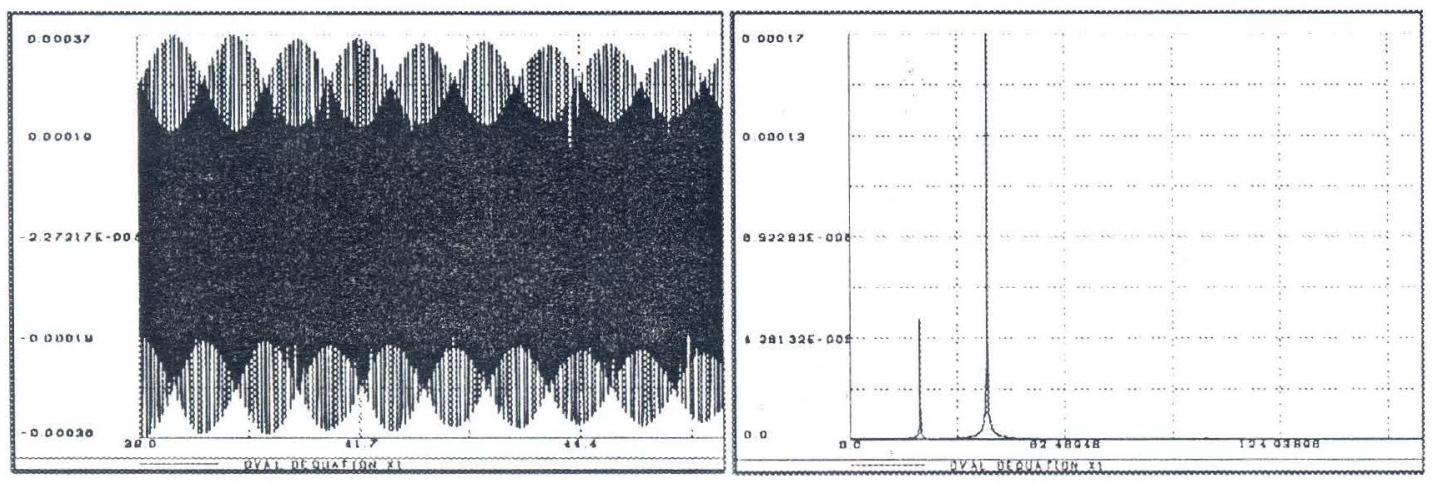

Fig. 13. Vibration $X_{1}$ and its spectrum for the shaft without parametric excitation $(\varepsilon=0)$ 


\subsection{External forces}

In this case, the dynamic loads applied on the system are similar as previous sections, but here the force in right hand of the first equation (5.4) is centrifugal force $F_{I}(t)$ (given by (3.5)) and the forces in right hand of the second equation (5.4) are:

$$
F(t)=F_{v 1}(t)+F_{v 2}(t)+F_{v 3}(t)
$$

where the right forces are given by formulae (3.6) - (3.8).
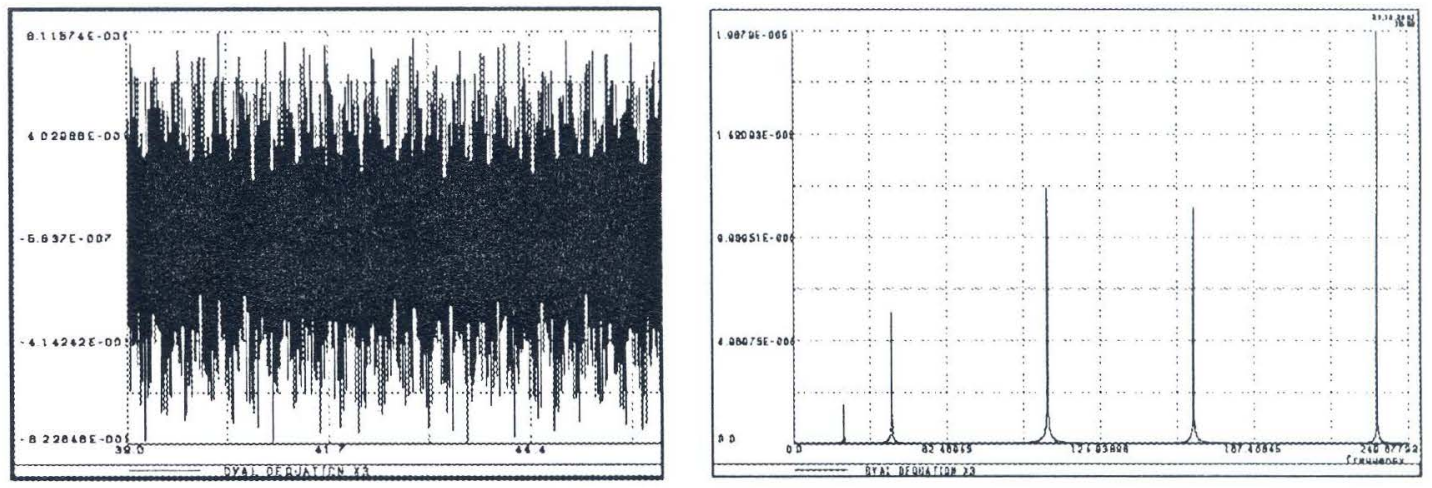

Fig. 14. Vibration $X_{2}$ and its spectrum for journal with the inner race in the case $\varepsilon=0$

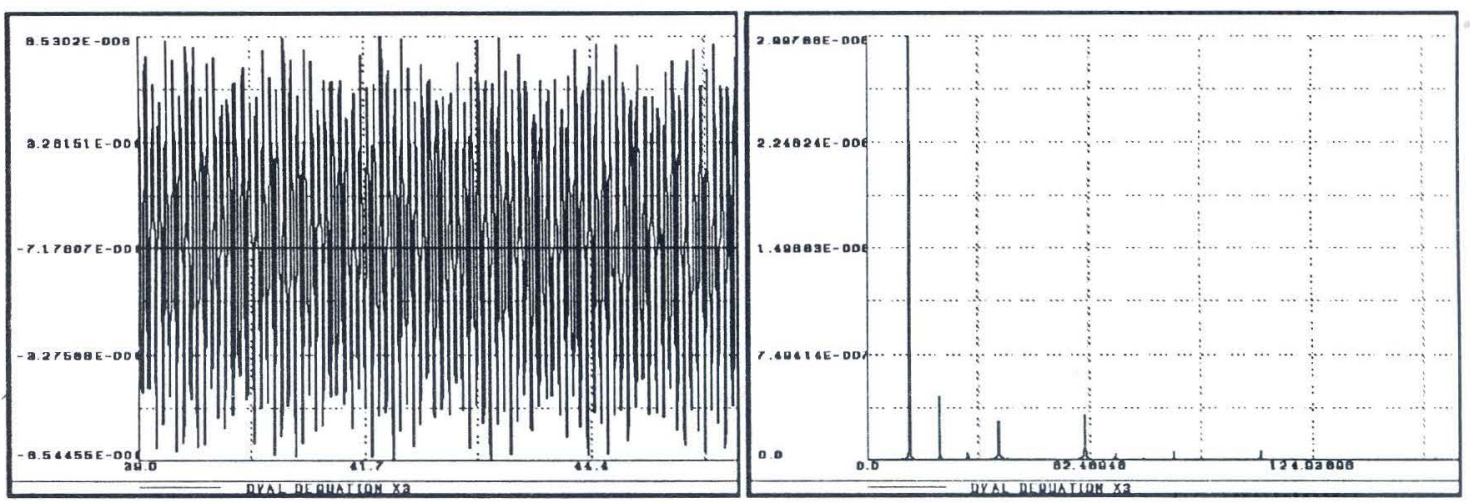

Fig. 15. Vibration $X_{2}$ and its spectrum when $f=12.08 \mathrm{~Hz}, \varepsilon=0$

\subsection{Some results of calculation}

Let us consider the system, in which there are a Jeffcott rotor supported by two ball-bearings of type 36212 (Fig. 11), with following parameters:

- Mass of the rotor $\mathrm{M}=1140(\mathrm{~kg})$.

- Mass of two inner races and journals $\mathrm{m}=10(\mathrm{~kg})$

- Pitch diameter $\mathrm{D}=2 \mathrm{R}=85.1(\mathrm{~mm})$

- Ball diameter $\mathrm{d}=2 \mathrm{r}=15.88(\mathrm{~mm})$

- Distance between two bearings: $\mathrm{L}=1(\mathrm{~m})$ 
- Diameter of the shaft $D_{1}=2 r_{1}=0.067(\mathrm{~m})$

- Area inertial moment of shaft cross section. $J=\left(\pi D_{1}^{4}\right) / 32=1.978 \times 10^{-6}\left(\mathrm{~m}^{4}\right)$,

- Unbalance mass $m_{0}=0.5(\mathrm{~kg})$

- Unbalance mass eccentricity $r=0.4(\mathrm{~m})$

- Number of balls: $N=10$

- Rotating speed: $f=12.08(\mathrm{~Hz})$ (=750 Rev./min)

- Damping coefficient: $d_{s}=(0.1) \mathrm{M}$

- Damping coefficient: $2 d_{B}=(0.3) \mathrm{M}$

- Small parameter $\varepsilon=0.3$

On the basis of previous formulae it is able to calculate coefficients and solutions of equation (5.4) for some cases.

1) There is no parametric excitation $(\varepsilon=0)$ in the equation system (5.4), but exist centrifugal force $F_{1}(t)$ and $F(t)=F_{v 1}(t)+F_{v 2}(t)+F_{v 3}(t)$.

The solution of equation (5.4) is given in the Fig.13, where on the left there is vibration of rotor shaft $\mathrm{x}_{1}$ and on the right is its spectrum. There are only two peaks of spectrum at centrifugal excitation frequency (the bigest) and first natural frequency (a smaller). But in the Fig.14, given vibration $\mathrm{x}_{2}$ and its spectrum of journal (with inner race), there are five peaks in spectrum at natural frequency and at frequencies of excitations produced by unbalance mass, and defects of inner race, outer race and breaking ball (Table 3 ).

Table 3.

\begin{tabular}{|c|c|c|c|c|c|}
\hline Frequency & 20.65 & 40 & 103 & 162,6 & 237 \\
\hline Intensity & $1.8 \mathrm{E}-6$ & $6.2 \mathrm{E}-6$ & $1.2 \mathrm{E}-5$ & $1.13 \mathrm{E}-5$ & $1.98 \mathrm{E}-5$ \\
\hline
\end{tabular}

Then if we can measure the vibration of journal at bearings, on the basis of spectral analysis it is able to show the types of defects in the ball bearings.

Notice that if there exists parameteric excitation with $\varepsilon$ sufficiently small (for example $\varepsilon=0.05$ ) then the vibration spectrum $\mathrm{x}_{2}$ is not much different from the Fig. 14, that means we can use the results as in the case $\varepsilon=0$.

2) There are centrifugal force $F_{1}(t)$ with frequency $f=12.08 \mathrm{~Hz}(750 \mathrm{rev} / \mathrm{min})$ and parametric excitation with $\varepsilon=0.3$, but $F(t)=0$ in the equations (5.4).

In this case, the vibration $x_{1}$ and its spectum are similar as on the Fig. 13 with two peaks at frequency $f=12.08 \mathrm{~Hz}$, and at first natural frequency $f_{n}=20.65 \mathrm{~Hz}$. 'The vibration $\mathrm{x}_{2}$ and its spectrum are expressed in the Fig.15 and the peaks of spectrum is given in Table 4.

Table 4. Parametric excitation frequency $f_{p}=49.12 \mathrm{~Hz}$

\begin{tabular}{|c|c|c|c|c|}
\hline Frequency & $\mathrm{f}=12.08$ & 20.65 & 37.0 & 61.16 \\
\hline Intensity & $3 . \mathrm{E}-6$ & $4.5 \mathrm{E}-7$ & $2.7 \mathrm{E}-7$ & $3.2 \mathrm{E}-7$ \\
\hline
\end{tabular}

On the basis of calculation, the parametric excitation frequency is $49.12 \mathrm{~Hz}$, then we have a remark from the table 4 that besides two peaks with frequencies $f$ and $f_{n}$, there are two other frequencies $37.0 \approx 49.12-12.08$, and $61.16 \approx 49.12+12.08$, that means it exposes the influence of parametric excitation. For belief of the previous remark, we take $f=16.5 ; 30$ and $40 \mathrm{~Hz}$ and get the following results (Table 5). 
Table 5.

\begin{tabular}{|c|c|c|c|c|c|}
\hline$f_{p}=67.1$ & Freq. peak & $\mathbf{1 6 . 5}$ & 20.65 & 50.04 & 83.11 \\
\hline & Intens. & $\mathbf{9 . 7 E - 6}$ & $1.2 \mathrm{E}-6$ & $1.1 \mathrm{E}-6$ & $1.5 \mathrm{E}-6$ \\
\hline $\mathrm{f}_{p}=122$. & Freq. peak & 20.65 & $\mathbf{3 0 .}$ & 91.68 & 151.28 \\
\hline & Intens. & $2.1 \mathrm{E}-6$ & $\mathbf{9 . 9 E - 6}$ & $1.4 \mathrm{E}-6$ & $1.5 \mathrm{E}-6$ \\
\hline $\mathrm{f}_{p}=162.67$ & Freq.peak & 20.65 & $\mathbf{4 0 .}$ & 121.89 & 202.31 \\
\hline & Intens. & $2.0 \mathrm{E}-6$ & $\mathbf{6 . 6 E}-\mathbf{6}$ & $1.2 \mathrm{E}-6$ & $9.6 \mathrm{E}-7$ \\
\hline
\end{tabular}

Check results in the Table 5 as previous remark, the frequencies in two final columns are approximately equal $f_{p}-f$ and $f_{p}+f$, the vibration $x_{2}$ and its spectrum for the case $f_{p}=122 \mathrm{~Hz}$ and $f=30 \mathrm{~Hz}$ are given in the Fig. 16 .
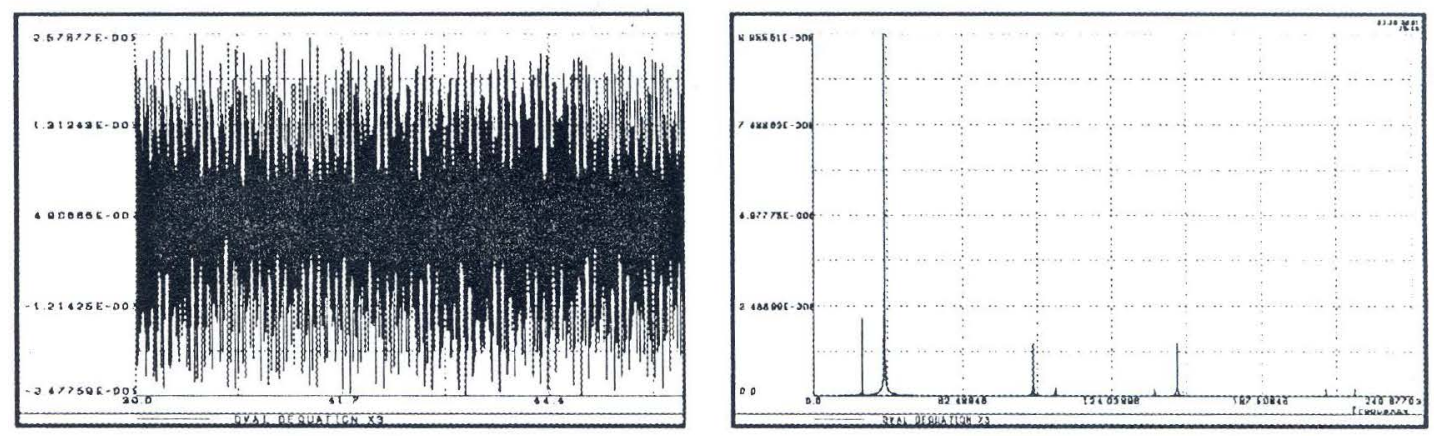

Fig. 16. Vibration $X_{2}$ and its spectrum when $f=30 \mathrm{~Hz}, f_{p}=122 \mathrm{~Hz}$

3) All forces produced by unbalance mass and defects of inner race, outer race and breaking ball are applied together to the system with parametric excitation $(\varepsilon \neq 0)$

In this case we take $f=40 \mathrm{~Hz}, \varepsilon=0.2$, and the frequencies are calculated and obtained $f_{i}=237 \mathrm{~Hz}, f_{e}=f_{p}=162.67 \mathrm{~Hz}, f_{b}=103 \mathrm{~Hz}$. The result of calculation is given in the Fig. 17 for vibration $x_{2}$ and its spectrum. In graphics of vibration spectrum there are some peaks besides excitation frequencies because of parametric excitation (Table 6).

Table 6 .

\begin{tabular}{|c|c|c|c|c|c|c|c|c|}
\hline Freq. & 20.65 & 40. & 59 & 74.5 & 103 & 162.67 & 174 & 237 \\
\hline Intens. & $1.9 \mathrm{E}-6$ & $6.3 \mathrm{E}-6$ & $1.5 \mathrm{E}-6$ & $1.3 \mathrm{E}-6$ & $1.2 \mathrm{E}-5$ & $1.1 \mathrm{E}-5$ & $1.2 \mathrm{E}-6$ & $2.0 \mathrm{E}-5$ \\
\hline
\end{tabular}

Notice that, besides domination frequencies of excitation forces, other peaks of spectrum gives the informations of ball-bearing quality as remark in the case 2). In this case, the vibration measurement data $\mathrm{x}_{1}$ of rotor shaft (not at bearings) can not be used for estimation of ball-bearing quality.

\section{CONCLUSION}

For assessinent of the ball-bearing quality by using data of vibration measurement, the three mechanical models for simulation of the rotor-ball bearing systems are proposed. 


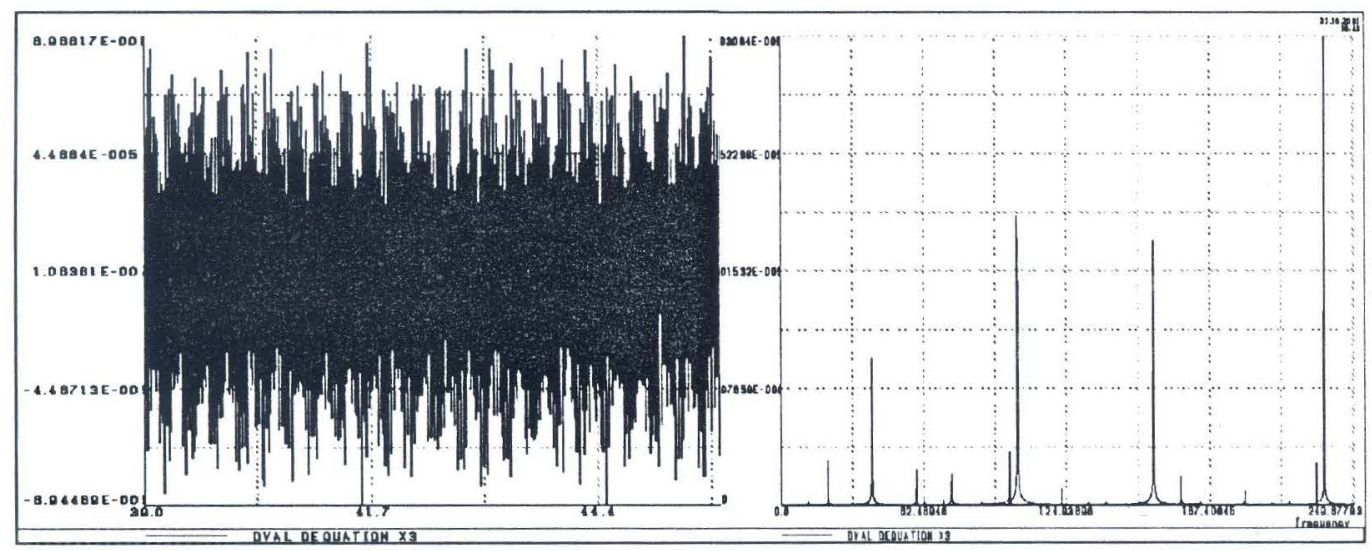

Fig. 17. Vibration and its spectrum for the case 3)

They are non-linear systems with parametric excitation and subjected to centrifugal force and impact forces produced by defects of ball-bearing (for example, inner race, outer race, breaking ball,...). If vibrations of rotor-bearing system are measured by eddy current proximity probe, that means, they are relative vibrations between journal and bearing cage, the models of simulation are single-degree-of-freedom system for rigid rotor and two-degree-of-freedom system for Jeffcott rotor. If vibrations of rotor-bearing system are measured on non-rotating parts by accelerometers then the modelling of the rotor-bearing system is two-degree-of-freedom system. After simulation of the systems and solving differential equations, the vibrations of the systems are obtained and analysed in the form of spectral density functions. their peaks are symptoms for identification and assessment of the ball bearing quality.

Acknowledgment. the publication is completed with financial support from the Council for Natural Science of Vietnam

\section{REFERENCES}

1. A.I Sprishevski, Rotating bearings (in Russian), Moscow 1969.

2. V. Wowk, Machinery Vibration, Measurement and Analysis. Mc. Graw Hill 1991.

3. Erwin Kramer, Dynamics of Rotors and foundations, Springer- verlag 1993.

4. Nguyen Cao Menh, A modelling for Simulating Vibrations of Bearing-Rotor systems, Int. Symposium on Dynamics and Control Hanoi, September15-17, 2003.

5. Nguyen Cao Menh and Vu Thanh Truc, Some symptoms for Diagnostics of ball- bearing quality, Second National Conference on Mechatronics, May-2004. Hochiminh city (in Vietnames).

6. Nguyen Cao Menh, Analysing vibrations of Rotor-Bearing-Gear box Systems, National Conference on Mechanics for $25^{- \text {th }}$ Anniversary of Institute of Mechanics, April 2004, Hanoi (in Vietnames).

7. Nguyen Cao Menh, Do Tran Thang, On the technical diagnosis of ball-bearings by vibration measurement on non-rotating parts $5^{\text {th }}$ ASEAM October, $5^{\text {th }}$, 2005. Hanoi

8. Nguyen Cao Menh, On the Simulation and Assessment for Vibration of Jeffcott rotor-ball bearing systems, Int. Conference on Nonlinear Analysis \& Engineering Mechanics today, December 11-14, 2006, Hochiminh city. 


\section{VỀ CHẨN ĐOÁN KỸ THUẬT CƯA Ổ BI DỰA TRÊN MÔ HÌNH Hệ RÔ TO - GỐI DỠ BẰNG PHƯƠNG PHÁP DAO DỘNG}

Trong bài này, sau khi phân tích biến dạng của ổ bi chịu tải, ta có thể khảo sát dao động của hệ rô to - gối đỡ như hệ với độ cứng phi tuyến và biến thiên tuần hoàn theo thời gian. Mô hình của hệ này phụ thuộc vào tính chất của của trục (cứng hoặc mềm) và vào phương pháp đo dao động của hệ (bởi đầu đo dòng xoáy không tiếp xúc với trục quay hay bởi đầu đo gia tốc trên phần không quay của hệ), nó có thể là hệ phi tuyến một bậc tự do hoặc hai bậc tự do chịu tác động của kích động tham số và lực ngoài. Do đó có thể nhận được những đặc tính khác nhau đối với dao động của hệ tương ứng với các khuyết tật khác nhau của ổ bi. Các dấu hiệu này giúp chúng ta nhận dạng và đánh giá chất lượng của ổ bi bằng việc đo và phân tích dao động của hệ. 